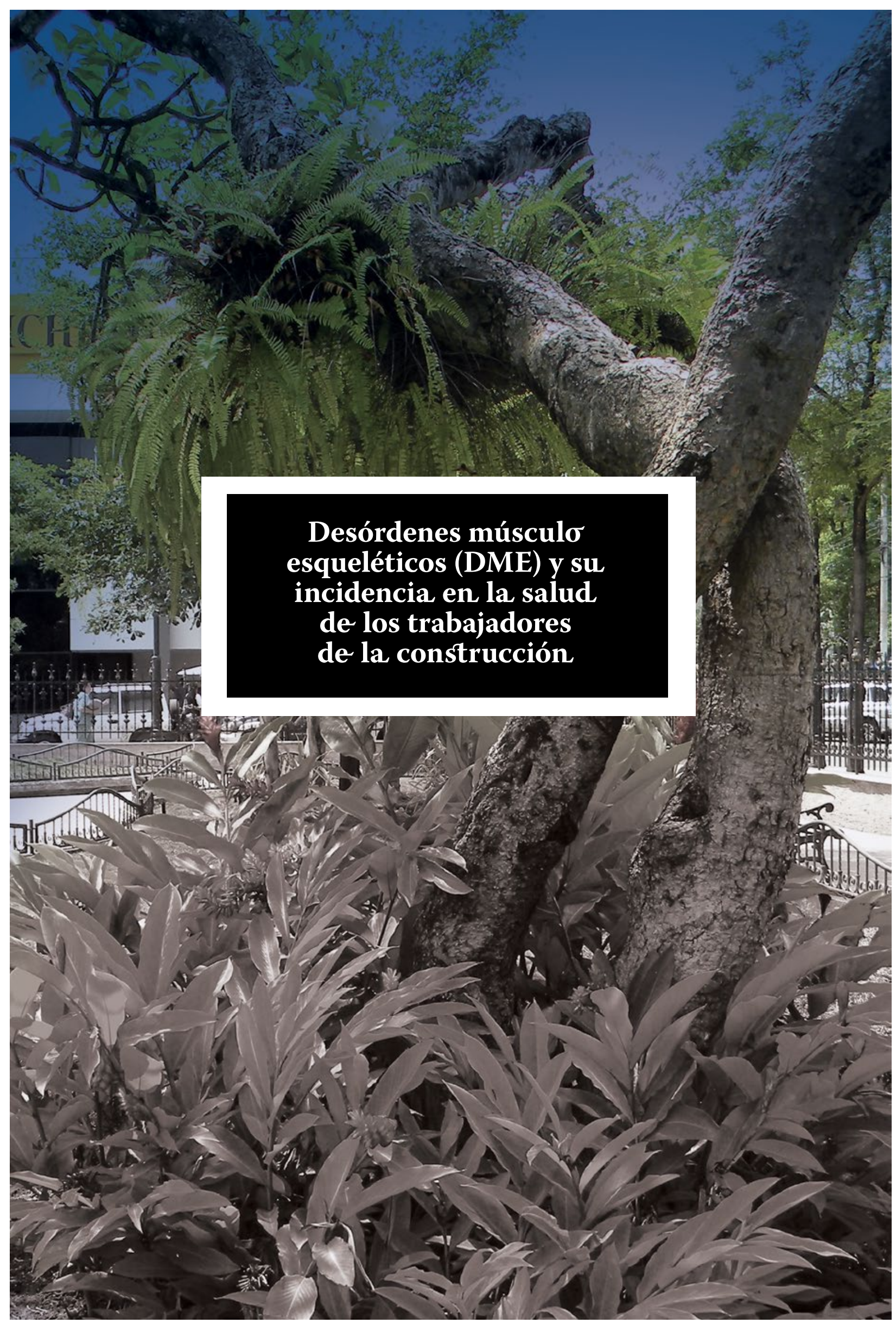




\title{
DESÓRDENES MÚSCULO ESQUELÉTICOS (DME) Y SU INCIDENCIA EN LA SALUD DE LOS TRABAJADORES DE LA CONSTRUCCIÓN
}

\section{MUSCLE SKELETON DISORDERS (DME) AND ITS INCIDENCE ON THE HEALTH OF WORKERS OF CONSTRUCTION}

\begin{abstract}
RESUMEN
Los desórdenes músculo esqueléticos (DME) relacionados con el trabajo son la causa principal de lesiones no fatales en la construcción, implican tensiones del sistema musculoesqueléticos del trabajador, en músculos, tendones, ligamentos, huesos, lo que ocasiona la disminución de su capacidad para ejecutar una actividad y el deterioro de la salud. Se revisó la evidencia disponible en medios electrónicos de información científica sobre la problemática de los DME en las actividades del sector de la construcción. Se evaluaron publicaciones con los criterios de inclusión y exclusión de evidencia científica en bases de datos como SciELO, RedAlyC, SCOPUS y/o MEDLINE, con publicaciones entre 2008 y 2018, en idioma español e inglés. La investigación aportó que los DME son la acumulación de traumas por exceso de acciones que atrofian segmentos corporales, prevenibles con características individuales, diseño de los puestos de trabajo y factores moduladores del riesgo que son elementos asociados a aspectos físicos, sicológicos y sociales, que se encuentran en la jornada laboral, el descanso, la contratación y la remuneración. El aporte de esta investigación a la comunidad científica se enfoca en acciones para minimizar los DME en trabajadores de la construcción en nuestro contexto.
\end{abstract}

PALABRAS CLAVE: Desórdenes músculo esqueléticos; seguridad y salud en la construcción; trabajadores de la construcción.

Copyright @ Revista San Gregorio 2019. ISSN 1390-7247; eISSN: 2528-7907 @

\begin{abstract}
The musculoskeletal disorders (MSD) related to work are the main cause of non-fatal injuries in construction, they involve tensions of the musculoskeletal system of the worker, in muscles, tendons, ligaments, bones, which causes the decrease of his ability to execute an activity and the deterioration of health. We reviewed the evidence available in electronic media of scientific information on the problem of SMEs in the activities of the construction sector. Publications were evaluated with the inclusion and exclusion criteria of scientific evidence in databases such as SciELO, RedALyC, SCOPUS and / or MEDLINE, with publications between 2008 and 2018, in Spanish and English. The research contributed that DME is the accumulation of traumas due to excess actions that atrophy body segments, preventable with individual characteristics, design of jobs and risk modulating factors that are elements associated with physical, psychological and social aspects, which find in the working day, rest, hiring and compensation. The contribution of this research to the scientific community focuses on actions to minimize DME in construction workers in our context.
\end{abstract}

KEY WORDS: Skeletal muscle disorders, safety and health in construction, construction workers.

Copyright @ Revista San Gregorio 2019. ISSN 1390-7247; eISSN: 2528-7907 @

José Vicente García Zambrano

Graduado de Maestría de Seguridad y Salud Ocupacio-

nal "Segunda Cohorte" de la Universidad San Gregorio

de Portoviejo. Manabí. Ecuador

$\sim$

vincen804@hotmail.com

ARTÍCULO RECIBIDO: 26 DE ABRIL DE 2019

ARTÍCULO ACEPTADO PARA PUBLICACIÓN: 12 DE JUNIO DE 2019

ARTÍCULO PUBLICADO: 30 DE JUNIO DE 2019 


\section{INTRODUCCIÓN}

Los riesgos del trabajo en la construcción, se relacionan con la demanda física de la tarea a realizar, con posturas inadecuadas, movimientos repetitivos, duplicaciones en las tareas, cargas estáticas y dinámicas, influenciado por falta de conocimiento de los trabajadores que adoptan posiciones no adecuadas, el tiempo que ésta se mantiene, la fuerza desarrollada y los movimientos pueden ser las causas de numerosos desórdenes músculo esqueléticos (Gonzáles, Bonilla, Quintero, Reyes \& Chavarro, 2016).

La construcción es valorada como una industria de alto riesgo debido a las características especiales que en ella se presentan. Los trabajadores de este sector son quienes muestran uno de los índices más altos de morbilidad, accidentabilidad y absentismo por enfermedades comunes, laborales y accidentes de trabajo (González, Fernández, Ballestero \& Quintana, 2015).

En estudios mundiales, se registra que los desórdenes músculo esquelético (DME) están en los primeros lugares de frecuencia en las patologías de origen ocupacional, relacionadas con índices altos de ausentismo laboral y costos elevados en las atenciones secundaria y terciaria (Martínez, Beltrán \& Patiño, 2014).

Según Mosquera (2012) en el Ecuador, los DME, son la causa principal de ausentismo laboral.

Los DME son una gran variedad de lesiones o trastornos de los músculos, los nervios, los tendones, los ligamentos y las articulaciones, principalmente en el miembro superior y la columna vertebral, la comunidad científica ha identificado que la aparición de los DME está asociada a la complejidad de los eventos como: dificultad para la manipulación de las cargas, movimientos pequeños en ciclos menores a dos minutos durante toda la jornada y postu- ras incómodas mantenidas por largo tiempo en la jornada de trabajo (Ramírez, 2012). Para determinar la presencia de la problemática se necesita la exposición clínica y el manejo médico depende de la estructura afectada. (Mendinueta, Herazo \& Pinillos, 2014).

Según la Organización Internacional del Trabajo (OIT) cada año se reportan alrededor de 160 millones de casos nuevos de enfermedades profesionales no mortales, con costos enormes para los trabajadores y sus familias, que están directamente ligadas a los DME (Moore, Aristizábal \& Velásquez, 2012) lo cual repercute en el desarrollo económico y social de los países; esta organización estima que los accidentes y las enfermedades profesionales originan la pérdida del $4 \%$ del producto interno bruto, es decir, cerca de 2,8 billones de dólares, en costos directos e indirectos (Barrero, Duarte, Vargas \& Villalobos, 2011); (Bonilla, González, Quintero \& Chavarro, 2016).

El presente trabajo científico es una revisión sistemática de la literatura, con el análisis del contenido guiada por las siguientes preguntas: ¿los factores asociados a aspectos físicos, sicológicos y sociales son causantes de DME en la construcción?, y ¿los DME son causados por acumulación de esfuerzo físico en las actividades constructivas?

Con el fin de hacer un aporte a la comunidad científica se revisa la evidencia de investigaciones científicas disponible en bases de datos, que contienen información de este tipo, sobre la problemática de los DME en las actividades laborales del sector de la construcción.

\section{METODOLOGÍA}

Se realizó la búsqueda de la literatura científica mediante revisión sistemática exploratoria (Manchado Garabito, 2009) que consisten en un mapeo de la literatura o evidencia científica sobre un tema en particular; para ello, fueron consultadas bases de datos internacionales de revistas científicas con acceso abierto como: Scielo, Redalyc, Scopus y/o Medline; se emplearon frases claves que formaron parte integral del desarrollo del presente artículo, las cuales fueron: Desórdenes musculoesqueléticos, Seguridad y salud en la construcción, y Trabajadores de la construcción. 
La revisión sistemática obtuvo su información de las fuentes académicas mencionadas con resultados transcendentales que tuvieron conclusiones de impacto; se utilizaron artículos científicos publicados entre los años 2008 y 2018, en idiomas español e inglés. Los resultados obtenidos respondieron a las variables: "Factores asociados a aspectos físicos, sicológicos y sociales son causantes de DEM en el trabajo"; y, "La acumulación de esfuerzo físico en las actividades constructivas es la causa principal de DME”. Se obtuvieron 2999 artículos de los cuales, tras una revisión primaria del título y año se escogen 1150. Tras un segundo análisis centrado en sus resúmenes se seleccionaron 163 publicaciones.

Finalmente, tras verificar que los artículos estuvieran disponibles en versión PDF en las bases de datos de acceso libre, el tamizaje redujo el número de publicaciones a 60 y tras un riguroso análisis de contenido se seleccionaron 36 artículos que cumplieron con la expectativa del investigador en función de las variables ya mencionadas y que son sujeto de la elaboración del presente artículo.

Los criterios de inclusión fueron: artículos originales y de revisión sistemática exploratoria, escritos en español e inglés, publicados en la última década, con énfasis en el continente americano, artículos que disponen textos completos; solo se seleccionaron artículos que contengan la frase desórdenes musculoesqueléticos. Los criterios de exclusión fueron aquellos que no eran pertinentes al tema tratado, estudios en cuyos objetivos de la publicación, no están los desórdenes musculoesqueléticos, y los trabajos de pregrado por no aportar información científica a la temática del estudio. Ver Figura 1 (En Anexos).

\section{RESULTADOS Y DISCUSIÓN}

De las publicaciones analizadas para elaborar el artículo de revisión sistemática exploratoria sobre la problemática de las DME, se obtuvo información acertada a nivel mundial y de países de la Región Andina que es cercano a nuestro medio, se presentan mediante la técnica de síntesis narrativa. Ver en la tabla No. 1 (En anexos) se muestra el origen de los artículos estudiados.

En el análisis de los artículos seleccionados para la revisión sistemática exploratoria (36 art.), se encontró que el 83,33\% de los escritos están en idioma español, el 16,67\% en idioma inglés. El panorama muestra que el $41,67 \%$ son artículos de revisión sistemática, el 58,33\% son artículos originales; cuantitativo cuasi experimental 13,88\%, el observacional descriptivo 8,33 El país de mayor aporte con información científica al apartado es Colombia con el $52,7 \%$, seguido por EEUU con el $16,7 \%$. Ecuador aportó con el 8,3\%, España con el $8,3 \%$ y los demás países con el 2,8\%.

Después del análisis y el criterio de los expertos se determinó que 9 artículos evaluaban diferentes exposiciones con resultados donde cinco de ellos eran transversales y cuatro de cohortes (Hauke, Flintrop, Brun \& Rugulies, 2011). De los documentos incluidos en el análisis de contenido, se determinaron que las estimaciones de riesgos agrupados y valores de heterogeneidad para cada subgrupo de estudios, se encontraron asociaciones estadísticas significativas, entre altas, demandas con la prevalencia de molestia y dolor en las extremidades superiores, molestia o dolor en cuello, molestia y dolor en espalda lumbar y molestia con dolor en extremidades inferiores, respectivamente (Seidel, Ditchen, Hoehne-Hückstädt, Rieger \& Steinhilber, 2019). Un control bajo sobre el trabajo tuvo asociación estadísticamente significativa con la prevalencia de molestia con dolor en cualquier región corporal. De la misma manera un apoyo social bajo en el trabajo de la construcción, se asoció a molestia o dolor en extremidades superiores, molestia con dolor en el cuello, molestia y dolor en cualquier región corporal. (Goode, Newnam, Van Mulken, Dekker \& Salmon, 2019).

La Organización Internacional del Trabajo (OIT) clasifica dentro del sector de la construcción a aquellas empresas públicas y privadas que erigen edificios para viviendas o fines comerciales e infraestructuras como carreteras, puentes, túneles, presas y aeropuertos (Bellorín, Sirit, Rincón \& Amortegui, 2018). Dentro de las funciones de los trabajadores de la construcción, se encuentra manipular cargas mayores a las permitidas, uno de los factores de riesgo que incide en la aparición de DME, lo cual evidencia que mientras más peso se debe levantar, más se incrementará el nivel de riesgo de desencadenar síntomas o DME de origen laboral del sector manifestado en las variables que rigen este estudio.

En el examen realizado a los artículos, en su análisis de contenido prevalece que la ma- 
yoría propone un panorama descriptivo de los DME definidos "como los problemas de salud del aparato locomotor, es decir, de músculos, tendones, esqueleto óseo, cartílagos, ligamentos y nervios pero no emite criterios que ayuden a eliminar o minimizar esta problemática influenciada por la cultura donde se desempeñan las actividades constructivas y las empresas que se dedican a dicha actividad (Cardeño, Portela \& Portela, 2015). De acuerdo al desempeño en la vida laboral del sector de la construcción, con los criterios de expertos y en función de los conocimientos adquiridos en la Maestría en Seguridad y Salud Ocupacional es la percepción de los autores que todo lo mencionado en la cita se agrava en la actualidad, porque las empresas que se dedican a esta actividad no acogen procedimientos de disminución de jornadas de trabajo, dando paso al sobresfuerzo de las extremidades superiores, por consecuencia afectación de los tendones y músculos. Lo expuesto es la opinión de un gran número de trabajadores de la construcción, con los que se tuvo oportunidad de interactuar durante el desarrollo de la investigación.

Por lo analizado sobre las enfermedades laborales en el sector de la construcción, se pudo notar que es donde se percibe un incremento de afectaciones en los trabajdores, por lo sensible de las condiciones de vida que muchos de ellos tienen y a las que se agrega las situaciones propias de la actividad laboral, las jornadas largas y exhaustivas y la inclemencia de los factores ambiéntales (Casallas \& Sánchez, 2015). Se añade que la bibliografía demuestra que el 92\% de los trabajadores de la construcción adoptan posturas inadecuadas, lo cual se manifiesta en la presencia de molestias en la zona del cuello y la espalda en más del 53\%, lo que provoca la aparición de diversas patologías (Castro et al., 2011). En la vida laboral los trabajadores que se dedican a las actividades constructivas, no cuentan con un régimen de capacitación y los centros donde se desenvuelven no cumplen características adecuadas para realizar la actividad profesional sin afectaciones a los músculos, tendones, ligamentos y miembros superiores.

Toda la evidencia estudiada a partir de la presente investigación expuesta y confrontada demuestra que existen factores que aportan a la aparición de los DME y que se debe buscar la manera de orientar su detección temprana y su prevención, desarrollando procedimien- tos con el único propósito de mejorar la seguridad y la salud en el trabajo (León \& Fornés, 2011); esta información de relevancia demostró que todos los avances que se señalan en el campo de la detección y prevención de los desórdenes músculo esquelético con relación a los factores de riesgo que se encuentran en las empresas y actividades constructivas de nuestro país no son las adecuadas para mejorar la calidad de vida de este sector de fuerza laboral que es muy deprimido.

Otros elementos presentes en las empresas del sector de la construcción, son los trabajos donde se debe aplicar mucha fuerza muscular, recibir vibraciones en una parte o todo el cuerpo, permanecer en espacios abiertos con mucha presencia de sol, los tiempos para cumplir con la tarea, la presión en el trabajo y/o los problemas que tienen fuera del trabajo, son factores asociados a la generación de DME propuesto por (Castro, 2016).

Se logró determinar que los trabajadores de la construcción están expuestos a demandas físicas exhaustivas, como lo es permanecer de pie por tiempos prolongados, adopción de posturas forzadas, realizar levantamiento de cargas manuales, así como la realización de movimientos forzados y repetitivos en las diferentes etapas del proceso constructivo. (Hernández, Ramírez \& Soto, 2016).

La supremacía en los resultados del 50\% de los estudios analizados, todos comparten el criterio de la prevalencia de una sintomatología alta de los desórdenes músculo esquelética y factores asociados; en cuello 54,3\%; seguida por, espalda alta 53,6\%; muñeca y mano $46,4 \%$ y columna lumbar 42\%; los mismos que se comparan con los presentados por Piedrahita (2014). Otro autor determinó que los dolores más frecuentes reportados por los trabajadores se encuentran en la región lumbar 71,5\%, el cuello 68\%, las manos y muñecas 58\% (Rodríguez, 2010). Todo esto por las condiciones laborales precarias que se presentan en nuestro medio sin protección alguna, sin derechos y legislación que los ampara para tener sueldos justos, áreas de trabajos acordes a sus condiciones, y sin daños psicológicos por presión de trabajar exhaustivas jornadas y sin reclamos porque, se pueden deslindar de sus servicios sin objeción y obtención de beneficios.

Sobre DME se confirma el criterio de que las variables estresores musculoesqueléticos 
causan alteraciones cuyos signos y síntomas pueden afectar distintas partes del cuerpo (mano, muñeca, codo, cuello y espalda) y diversas estructuras anatómicas (hueso, músculo, tendones, articulaciones y nervios) (Hernández, Ramírez \& Soto, 2016). El síntoma clave es el dolor, el cual es una sensación subjetiva que constituye muchas veces la única manifestación, este puede caracterizarse como agudo o crónico (Trillos, Tolosa \& Escobar, 2016) y es el síntoma más frecuente en la población mundial y es el único indicio para comenzar a tratar la problemática que presentan la mayoría de la mano de obra útil para la sociedad (Soto \& Muñoz, 2018), ya que ellos utilizan su atributo, como lo es la aplicación de la fuerza la misma que desencadena la tensión producida en los músculos por el esfuerzo requerido para el desempeño de una tarea, como levantar o manipular una carga, hacer un agarre, ejecutar una herramienta. Se presume que a mayor fuerza mayor grado de riesgo, especialmente en los grandes segmentos corporales como el hombro, el cuello y la espalda baja (Ordóñez, Gómez \& Calvo, 2016). Los mismos determinan que los Desórdenes Musculoesqueléticos son la acumulación de trauma por el exceso de acciones que atrofian algunos segmentos corporales, los cuales son totalmente prevenibles, tomando en cuenta las características individuales, el diseño de los puestos de trabajo y factores moduladores del riesgo como la jornada laboral, tiempo de descanso, tipo de contratación y la remuneración que es uno de los factores transcendentales.

La relación del estrés con el trabajo, están correlacionados por que los trabajadores expuestos a sesiones intensas provocan fatiga en el sistema osteomuscular, que su acumulación puede desencadenar problemas de ausentismo laboral (Salvador, 2018). Con el avance y el progreso de las investigaciones, varios autores determinan que en la actualidad por los adelantos tecnológicos es conocido que existe una relación directa entre el estrés y la aparición de los DME relacionados con el trabajo.

Los principales factores de riesgo relacionados con la aparición de los desórdenes musculoesqueléticos de cuello y extremidades superiores son los siguientes: aplicación de una fuerza que da lugar a una presión mecánica intensa en el cuello, hombro y extremidades superiores; trabajo en posturas forzadas, con contracción muscular continua y soporte de grandes cargas mecánicas; movimientos repetitivos, principalmente si involucran el mismo grupo muscular y articular (Bedoya, Osorio, Tovar, Roqueme \& Espinoza, 2018). Lo que demuestra que entre actividad muscular y repetición de trabajo prolongado sin pausas, ni descanso que faciliten la recuperación después de la manipulación de carga con presión directa sobre herramientas y equipos con vibración mano-brazo que producen entumecimiento, cosquilleo, o pérdida de la sensibilidad, que obliga a ejercer mayor presión de agarre, lo que conlleva a que los resultados proporcionen criterios solidos a la comunidad científica, con acciones correctivas para minimizar los desórdenes musco esqueléticos en los obreros que se dedican actividades de la construcción en nuestro medio.

De esta forma, se confirma según el Instituto Nacional para la Salud y Seguridad Ocupacional (NIOSH) que el costo asociado con los DME es muy alto: más de US\$ 2,1 billones en compensaciones y US\$ 90 millones en costos directos anuales. Asimismo, la incidencia se ha incrementado, donde se pasó de 141000 casos en 2012 a 184000 en 2013, se evidencio en el 2015 que estos trastornos se consideran la nueva epidemia de enfermedades crónicas en el mundo actual y su origen es multifactorial (González, Bonilla, Quintero, Reyes \& Chavarro, 2016) por lo que se han abordado de manera interdisciplinar en la búsqueda del máximo confort de las personas en sus estaciones de trabajo y el equilibrio entre el sistema hombre, máquina, sistema de trabajo y ambiente (Martínez Paéz, 2016).

La pérdida de dicho equilibrio puede ocasionar daños en la salud, entre ellos un índice alto de presentar molestias por DME, se debe enfocar una estrategia atractiva para la prevención osteomuscular a fin de intervenir sobre la exposición a riesgos ergonómicos en el trabajo en los países de renta baja como lo es ecuador (Gómez, Suasnavas, Vilaret, Silva \& Russo, 2016).

En la actualidad la única forma de aportar para mejorar la calidad de vida en la clase obrera que se dedican a la construcción, es replicar estudios de estas características para darles a conocer que se tiene que trabajar en acciones concretas, para obtener resultados que ayuden a mejorar la protección de la salud y seguridad de los trabajadores, ya que 
esto se logra a través de las áreas del conocimiento como lo es investigación y publicación para que sean replicados en el ámbito laboral de Manabí y Ecuador, ya que la mano de obra está expuesta a la problemática en estudio, donde se permita adecuar los ambientes de trabajo con los procedimientos en función de las capacidades de cada individuo.

Rojas, Gimeno, Vargas-Prada \& Benavidez (2015) en su estudio afirman que en la industria de la construcción los síntomas o desórdenes músculo esqueléticos están ampliamente distribuidos y se observan en trabajadores relativamente jóvenes. Por lo que constituyen la principal causa de incapacidad y pérdida de tiempo por enfermedad ocupacional para muchos grupos de mano de obra joven que ven diezmadas sus posibilidades de seguir activos en la oferta de servicios; y accesible a remuneraciones acordes al mercado, siendo este una hegemonía en la población trabajadora tanto en países desarrollados como en vías de desarrollo.

No se encontró relación significativa entre los Desórdenes musculoesqueléticos y la edad ya que el mayor porcentaje de casos se ubicó en trabajadores jóvenes, destacando el grupo de 30 a 39 años, donde se encuentra la mayor frecuencia de trabajadores, así mismo, se observa un descenso de los mismos a partir de los 40 años de edad (Rodríguez \& Dimate, 2015). Esto podría deberse a que los trabajadores que realizan una determinada labor, a través de los años aprenden y desarrollan estrategias para realizar sus tareas con menor riesgo, así mismo la continuidad y antigüedad laboral los coloca en otros cargos que ameritan menor esfuerzo físico y cuentan con apoyo de otros trabajadores, disminuyendo de esta manera la posibilidad de presentar patologías músculo esqueléticas.

Estos resultados de este estudio coinciden con otras investigaciones, donde el mayor número de casos fue reportado en trabajadores jóvenes, en los cuales no se encontró igualmente asociación entre la edad y la presencia de síntomas músculo esquelético (Escudero-Sobogal, 2017). Las nuevas formas de organización del trabajo provocan incertidumbre, precarización y violación de los derechos laborales de los trabajadores de la construcción, más aún que es un sector vulnerable porque tiene que aceptar condiciones de trabajo muy precarias y riesgosas para poder sobrevivir.
Las jornadas extenuantes (por intensificación y alarga miento de la jornada) son un punto medular que es necesario modificar, pues además de que violan flagrantemente las leyes laborales del Ecuador. (Trillo et al., 2016). Las causas que han motivado este crecimiento tienen que ver con el auge de la actividad económica, creación, ampliación y modernización de empresas, mejora de las comunicaciones y el transporte, todo ello gracias a la fuerte demanda de obra civil y edificación.

La edad de los trabajadores y los desórdenes musculo esqueléticos se encuentran estrechamente ligados por la existencia de trabajos repetitivos con ciclos de trabajos cortos, posturas forzadas, carga física entre otros (Castro, Ardila, Orozco, Sepulveda \& Molina, 2018). Por lo anterior se puede concluir que en este estudio no se puede afirmar que la única asociación al desarrollo de DME sea la exposición al riesgo por tiempo prolongado, dado que los trabajadores no siempre tienen una antigüedad significativa en las empresas que tienen labores constructivas.

Lo que implica la aparición de desórdenes musculo esqueléticos por posturas forzadas y movimientos bruscos relacionados con la fuerza, que este es el factor más denotado por los autores en los diferentes artículos estudiados para la elaboración de la revisión sistemática ejecutada como investigación.

Con la aparición de DME en obreros que utilizan herramientas de la construcción, se encontró asociación estadísticamente significativa para hombro, espalda baja, antebrazo, cadera y pierna, estos resultados aseveran que los ciclos de trabajo y la repetitividad de tareas en las áreas de trabajo analizadas en el sector de la construcción tienen relación directa y son susceptibles de ser usadas para la evaluación de los DME de origen laboral (Dimate, Rodríguez \& Rocha, 2017). La propuesta de enfoque integrador puede ayudar en el desarrollo de estrategias de intervención para los DME, de origen laboral ya que las investigaciones que fueron sujeto de análisis aportan que el sector de la construcción ha sido el más dinámico en términos de creación de empleo, y con ellos jornadas largas de trabajo, que incrementan el riesgo laboral por diversas razones, como el cansancio por deshidratación, la falta de atención que pueden desencadenar la atrofia de los músculos, cartílagos y extremidades, pero todo ello se debe controlar, 
minimizar y eliminar para tener trabajadores productivos en las diferentes empresas y ser aporte para sus familias.

De igual manera en lo relacionado con los factores de riesgo de DME, el $70 \%$ relaciono significativamente la edad, el $60 \%$ la realización de movimientos repetitivos y el $58 \%$ la duración de la jornada laboral, dichas asociaciones se relacionan también con otros factores de riesgo encontrados como es estar sentado en posición incómoda, trabajar sin descanso alguno durante 10 horas o más, no tener un puesto de trabajo estable y riesgos organizacionales (Agila, Colunga, Gonzáles \& Delgado, 2014). Se encontró evidencia moderada del beneficio de cambios integrales en el puesto de trabajo de actividades formativas sobre los trabajadores y del uso de herramientas en rotación que permiten adoptar posturas más apropiadas de la extremidad superior y evitar molestias con daños en la salud de ellos.

Adicionalmente los factores individuales modificables deben ser seriamente contemplados en la prevención de los DME relacionados con el trabajo, ya que aspectos como el sobrepeso y el hábito de fumar son incluidos dentro de sus posibles causas, se propende el concepto de vida activa como una apuesta a la actividad física con el objetivo de mejorar la capacidad funcional de los trabajadores (Satizábal, 2011). Es así como se puede concluir, que quien realmente esté interesado en prevenir la aparición de los DME relacionados con el trabajo riguroso de la construcción, no puede limitar sus esfuerzos al control de la carga física, sino que debe contemplar todos los peligros asociados a su aparición, y como parte de este proceso desarrollar un ambiente participativo en el que tengan lugar las alternativas de solución propuestas por la voz de la población trabajadora.

Ésta es la interpretación que se debe elegir e impulsar si efectivamente existe una intención por controlar los factores de riesgo y sus actividades, disminuir el ausentismo, aumentar la productividad y mejorar las condiciones de trabajo y de vida al interior de las empresas constructoras (García, 2016) ya que los estudios obtuvieron resultados beneficiosos para la población intervenida, excepto en el caso de intervenciones sobre la organización del trabajo, que encontraron evidencia parcial de incrementos con prevalencia de dolor muscu- lo esquelético cuando los cambios introducidos van en detrimento de las condiciones psicosociales.

\section{CONCLUSIONES}

- La revisión realizada corrobora que en la actualidad existe una tendencia a estudiar enfermedades relacionadas con los agentes físicos que a su vez se relaciona con sectores de gran demanda laboral como es el sector de la construcción por ejemplo (desórdenes músculo esquelético, afectaciones por ruido y vibraciones) por encima de agentes químicos o biológicos, por motivo de contar con herramientas aplicables al medio donde nos desenvolvemos.

- los desórdenes músculo esqueléticos representan una de las primeras causas de ausentismo laboral en las empresas y generan un gran impacto a nivel económico, social y humano en ecuador y el continente americano.

- Los factores moduladores del riesgo que son elementos asociados a aspectos físicos, sicológicos y sociales del entorno laboral que a la aparición de los DME producto de las actividades del sector de la construcción que en nuestro país no son estudiadas incidiendo en la falta de atención al sector vulnerable que son los trabajadores.

- El exceso de trabajo provoca errores en el desarrollo de sus acciones como son manipulación manual de cargas, posturas fijas y mantenidas por tiempos prolongados y los movimientos repetitivos, entre otros factores personales y del trabajo incidiendo en la aparición temprana de los DME de origen laboral de la construcción.

- En el progreso de las investigaciones varios autores determinan que en la actualidad con los avances tecnológicos es bien sabido que existe una relación directa entre el estrés y la aparición de los DME relacionados con el trabajo.

- La disminución de las capacidades de un trabajador de la construcción desencadena otras problemáticas sociales como es el despido de su puesto de trabajo, el cual representa un problema social porque es su única fuente de ingreso el cual afecta el entorno familiar, por estas razones se tiene que buscar meca- 
nismo orientados a la protección de este sector vulnerable social y laboralmente.

- Cabe mencionar que de la bibliografía recabada en ningún artículo se manifiesta una recuperación total de la capacidad de trabajar en el área de la construcción sin embargo en todos se observan resultados positivos en varios aspectos como son disminución importante del dolor expresada por el paciente o por la escala análoga visual, reincorporación a actividades funcionales del miembro lesionado y en otros se hicieron estudios radiológicos que confirmaron la recuperación de la lesión a nivel óseo, muscular o tisular.

\section{REFERENCIAS BIBLIOGRÁFICAS}

Agila, E., Colunga, C., Gonzáles, E., \& Delgado, D. (2014). Síntomas Músculo Esqueléticos en trabajadores Operativos del Área de Mantenimiento de una Empresa Petrolera Ecuatoriana. Ciencia y Trabajo, 15(51), 198205.

Ballester, A., \& García, A. (2017). Asociación entre la Exposición Laboral a Factores Psicosociales y la existencia de Trastornos Musculoesqueléticos en Personal de Enfermería: Revisión Sistemática Y Meta-Análisis. Rev Esp Salud Pública, 91(7), 4-28.

Barrero, L., Duarte, A., Vargas, A., \& Villalobos, G. (2011). Prevención de desórdenes musculoesqueléticos de las extremidades superiores relacionados con el trabajo: revisión sistemática. Archivos de prevención de riesgos laborales, 138-146.

Bedoya, E., Osorio, I., Tovar, C., Roqueme, K., \& Espinoza, E. (2018). Determinación de la carga física como factor de riesgo de desórdenes osteomusculares. ESPACIOS, 39(06), 10-23.

Bellorín, M., Sirit, Y., Rincón, C., \& Amortegui, M. (2018). Síntomas músculo esqueléticos en trabajadores de una empresa de construcción civil. Salud de los trabajadores, 12(4), 125-136.

Bonilla, J., González, A., Quintero, M., \& Chavarro, A. (2016). Análisis de las causas y consecuencias de los accidentes laborales ocurridos en dos proyectos de construcción. Revista ingeniería de construcción, 8(12), 05-16.

Cardeño, W., Portela, N., \& Portela, E. (2015). Desórdenes músculo esqueléticos en funcionarios administrativos usuarios de video terminales/skeletal muscle disorders in administrative personnel users of video terminals. Revista Ciencias de la Salud, 10(2), 78-91.

Casallas, M. \& Sánchez, F. (2015), Los costos de la enfermedad laboral: revisión de literatura. Facultad Nacional de Salud Pública. El escenario para la salud pública desde la ciencia. Revista Ciencias de la Salud, 33(2), 218-227.

Castro, C. (2016). Diseño de sistema de vigilancia epidemiológica en desórdenes osteomusculares para una empresa de fabricación de refrigeradores en el distrito de Barraquilla. Biociencias, 11(1), 15-28.

Castro, E., Múnera, J., Sanmartín, M., Valencia, N., Valencia, N., \& González, P. (2011). Efectos de un programa de pausas activas sobre la percepción de desórdenes músculo-esqueléticos en trabajadores de la Universidad de Antioquia. Educación física y deporte, 30(1), 389-399.
Castro, G., Ardila, L., Orozco, Y., Sepulveda, E., \& Molina, C. (2018). Factores de riesgo asociados a desordenes musculo esqueléticos en una empresa de fabricación de refrigeradores. Rev. Salud Pública, 20(2), 182-188.

Dimate, A., Rodríguez, D., \& Rocha, A. (2017). Percepción de desórdenes musculoesqueléticos y aplicación del método RULA en diferentes sectores productivos una revisión sistemática de la literatura. Rev Univ Ind Santander Salud, 49(1), 57-74.

Escudero-Sobogal, I. (2017). Síndrome del túnel carpiano como desorden musculoesquelético de origen laboral. Revista Libre Empresa, 14(2), 229235.

García, A. (2016). Propuesta metodológica para el establecimiento de líneas de investigación en seguridad de los accidentes laborales ocurridos en dos proyectos de construcción. Revista ingenieria de construcción, 8(5), 05-16.

Gómez, A., Suasnavas, P., Vilaret, A., Silva, M., \& Russo, M.(2016).Propuesta metodológica para el establecimiento de líneas de investigación en seguridad y salud en el trabajo. INNOVA Research Journal, 1(9), 13-25.

Gonzáles, A., Bonilla, J., Quintero, M., Reyes, C., \& Chavarro, A. (2016). Análisis de las causas y consecuencias de los accidentes laborales ocurridos en dos proyectos de construcción. Revista ingeniería de construcción, 31(1), 05-16.

González, A., Bonilla, J., Quintero, M., Reyes, C., \& Chavarro, A. (2016). Análisis de las causas y consecuencias de los accidentes laborales ocurridos en dos proyectos de construcción. Ingeniería de construcción RIC, 31(1), 0516.

González, I., Fernández, M., Ballesteros, J., \& Quintana, S. (2015). Ruido y vibraciones en el sector de la construcción. Rev. Salud Pública., 4(7), 3645.

Goode, N., Newnam, S., Van Mulken, M., Dekker, S., \& Salmon, P. (2019). Investigating work-related musculoskeletal disorders: strengths and weaknesses of current practices in large Australian organizations. Safety Science. Safety Science, 1(12), 105-115.

Hauke, A., Flintrop, J., Brun, E., \& Rugulies, R. (2011). The impact of work-related psychosocial stressors on the onset of musculoskeletal disorders in specific body regions: A review and meta-analysis of 54 longitudinal studies. Work y Stress, 25(3), 243-256.

Hernández, A., Ramírez, G., \& Soto, A. (2016). Desórdenes musculo esqueléticos relacionados con el trabajo. Revista Colombiana de Salud Ocupacional, 6(1), 27-32. 


\section{REFERENCIAS BIBLIOGRÁFICAS (CONT.)}

Hernández, A., Ramírez, E., \& Soto, A. (2016). Desordenes músculo esquelético relacionados con el trabajo. Revista colombiana de salud ocupacional, 6(1), 27-32.

León, M., \& Fornés, J. (2011). Estrés psicológico y problemática de los desórdenes musculoesqueléticos revisión sistemática. Enfermería Global. Revista Colombiana de Salud, 8(12), 276-300.

Manchado Garabito, R. T. (2009). Revisiones sistemáticas exploratorias. Medicina y seguridad del trabajo, 55(216), 12-19.

Martínez Paéz, J. (2016). Condiciones de trabajo en sector de la construcción: una revisión de la literatura en el periodo comprendido del 2000 al 2015. Revista de la Construcción, 2(6), 33-45.

Martínez, M., Beltrán, Y., \& Patiño, Y. (2014). Factores asociados a la percepción de dolor lumbar en trabajadores de una empresa de transporte terrestre. Salud Uninorte. Barranquilla (Col), 30(2), 192-199.

Mendinueta, M., Herazo, Y., \& Pinillos, Y. (2014). Factors associated with the perception of low back pain in workers of a ground transportation company. Revista Salud Univorte, 7(5), 192-199.

Moore, P., Aristizábal, J., \& Velásquez, J. (2012). Síntomas músculo esqueléticos y percepción de calidad de vida en salud en trabajadores de una curtiembre. Revista Colombiana de Salud ocupacional, 7(4), 11-15.

Mosquera, E. (2012). Seguridad y Salud en las obras de construcción. Ciencia y Trabajo, 1(3), Ministerio de trabajo y empleo del Ecuador 11-23.

Ordóñez, C., Gómez, E., \& Calvo, A. (2016). Desórdenes músculo esqueléticos relacionados con el trabajo. Revista Colombiana de Salud ocupacional, 6(1), 27-32.

Piedrahita, H. (2014). Evidencias epidemiológicas entre factores de riesgo en el trabajo y los desórdenes músculoesqueléticos. Revista MAPFRE Medicina. Revista Colombiana de Salud, 15(4), 212-221.

Ramírez Ortíz, J. (2012). Desórdenes músculo esqueléticos en miembros torácicos y factores de riesgo ergonómico en trabajadores con postura forzadas. Ciencia y Salud Pública, 5(7), 43-54.

Rodríguez, D., \& Dimate, A. (2015). Evaluación de riesgo biomecánico y percepción de desórdenes músculo esqueléticos en administrativos de una universidad Bogotá (Colombia). Investigaciones Andina, 17(31), 1284-1299.

Rodríguez, E. (2010). Protección de la seguridad y salud de los trabajadores. Una revisión desde la perspectiva global, latinoamericana y venezolana. Ingeniería Industrial. Actualidad y Nuevas Tendencias. Ingeniería Industrial. Actualidad y Nuevas Tendencias, II (5), 81-96.

Rojas, M., Gimeno, D., Vargas-Prada, S., \& Benavides, F. (2015). Dolor musculoesquelético en trabajadores de América Central: resultados de la I encuesta Centroamericana de Condiciones de Trabajo y Salud. Rev Panam Salud Pública, 38(2), 20-8.

Salvador., J. (2018). Riesgos Psicosociales del Sector Aeroportuario de Manta. Revista San Gregorio, 1390(7247), 31- 40.

Satizábal, J. (2011). Un enfoque holístico en la prevención de los desórdenes músculo esqueléticos relacionados con el trabajo. Revista Cubana de Salud y Trabajo, 12(3), 61-
Seidel, D., Ditchen, D., Hoehne-Hückstädt, U., Rieger, M., \& Steinhilber, B. (2019). Quantitative measures of physical risk factors associated with work-related musculoskeletal disorders of the elbow: systematic reviw. International journal of environmental research and public health, 16(1), 130-143.

Soto, F., \& Muñoz, C. (2018). Percepción del Beneficio del Ejercicio para la Prevención de Trastornos Musculoesqueléticos. Una Perspectiva del Trabajador. Cienc Trab, 20(61), 14-18.

Trillos, M., Tolosa, I., \& Escobar, R. (2016). Modelos de rehabilitación para la intervención del desorden músculoesquelético en trabajadores. Rev Univ Ind Santander Salud (4), 536-547. 


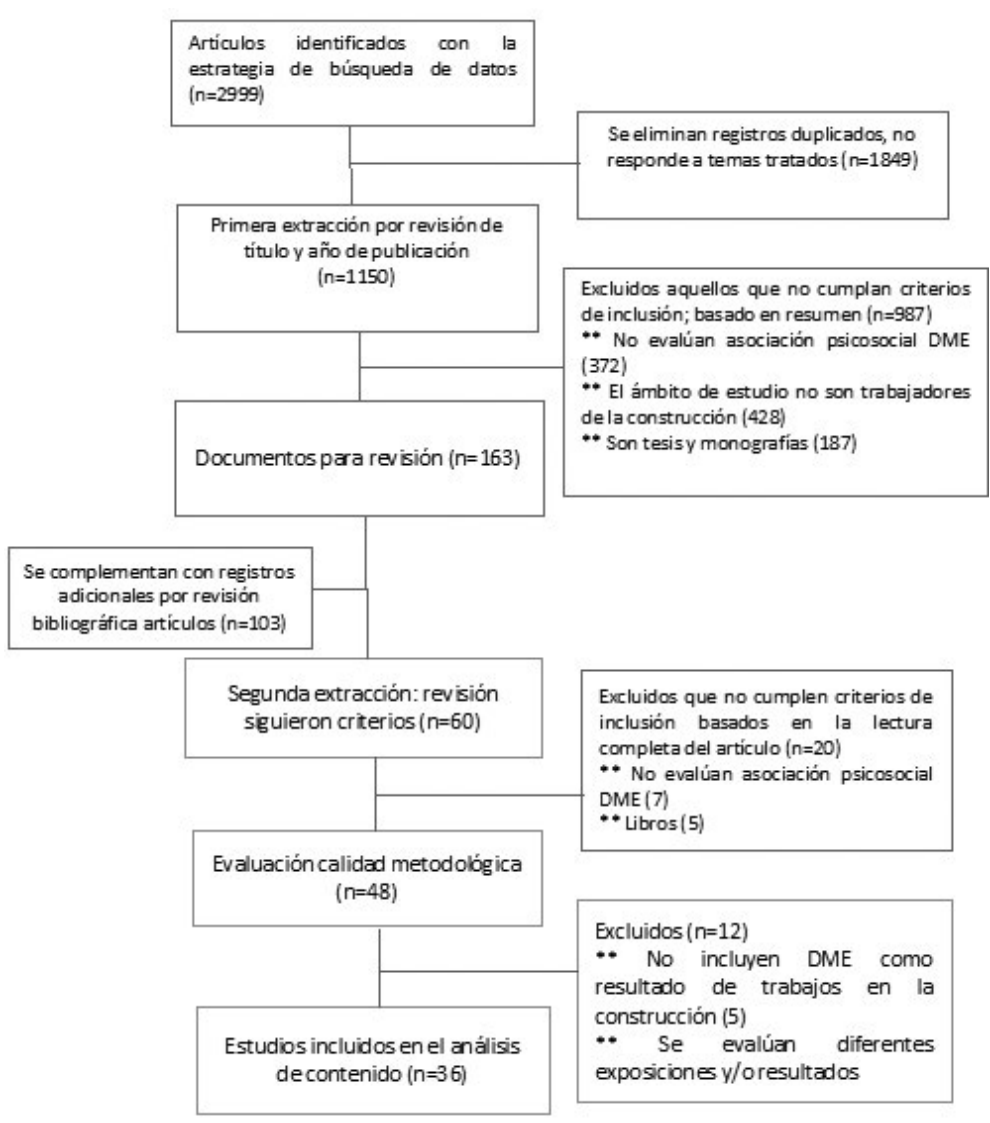

Figura 1. Diagrama del proceso de búsqueda bibliográfica y selección de trabajos Fuente: Según el esquema presentado por (Ballester E García, 2017) con adaptación y elaboración propia a partir de los datos obtenidos en la revisión sistemática exploratoria.

\begin{tabular}{|l|c|c|}
\hline \multicolumn{1}{|c|}{ País } & $\mathrm{N}^{\circ}$ de artículos & $\%$ \\
\hline Colombia & 19 & 52,7 \\
\hline Estados Unidos & 6 & 16,7 \\
\hline España & 3 & 8,3 \\
\hline Ecuador & 3 & 8,3 \\
\hline Perú & 1 & 2,8 \\
\hline Argentina & 1 & 2,8 \\
\hline Chile & 1 & 2,8 \\
\hline Panamá & 1 & 2,8 \\
\hline México & 1 & 2,8 \\
\hline
\end{tabular}

Tabla $N^{\circ}$ 1. Ubicación geográfica de los artículos analizados.

Fuente: Elaboración propia. 


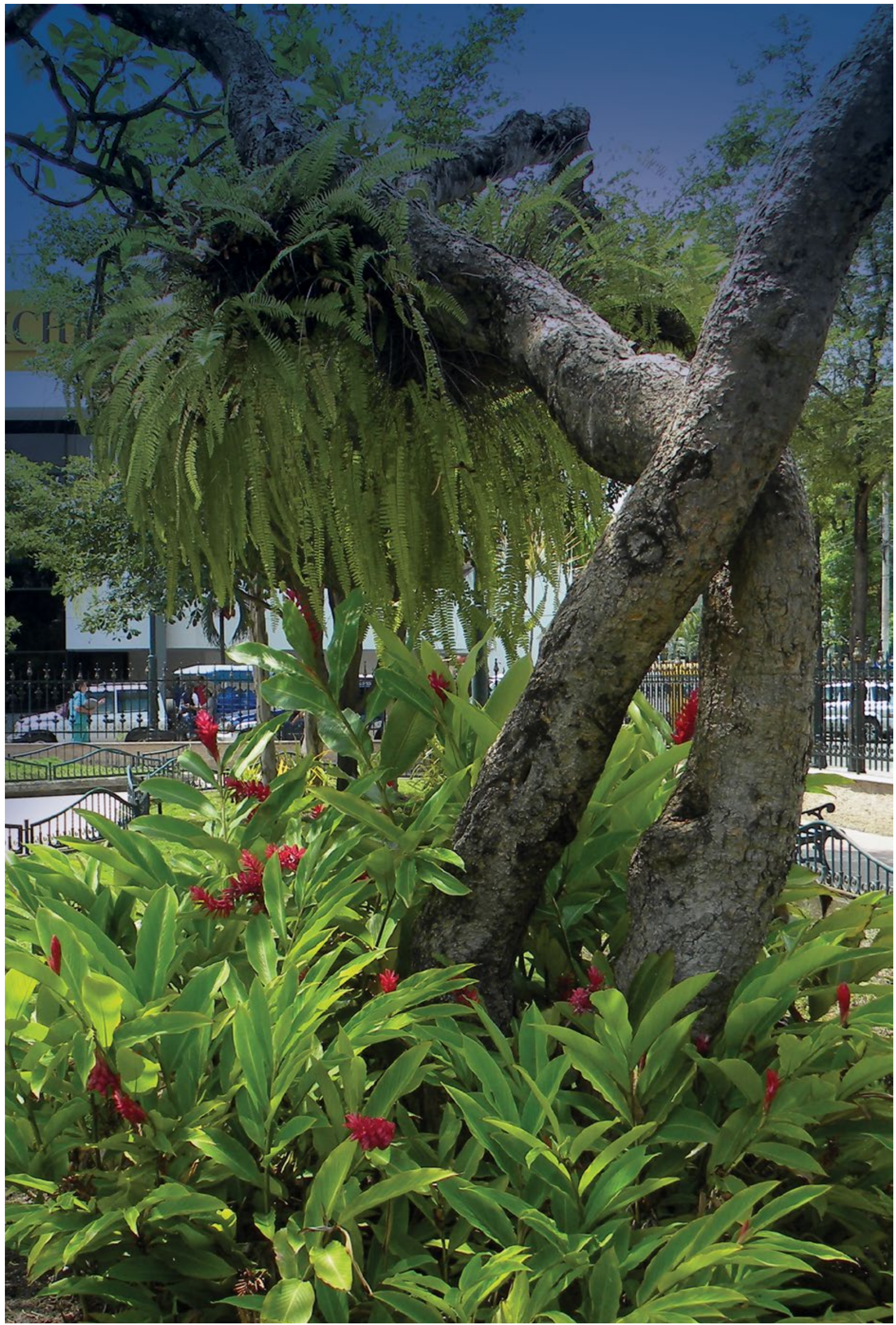

